NIIG-DP-01-03

September, 2001

hep-th/??????

\title{
Regularized Quantum Master Equation in the Wilsonian Renormalization Group
}

\author{
Yuji Igarashi, Katsumi Itoh and Hiroto $\mathrm{So}^{a}$ \\ Faculty of Education, Niigata University, Niigata 950-2181, Japan \\ a Department of Physics, Niigata University, Niigata 950-2181, Japan
}

\begin{abstract}
Using the Pauli-Villars regularization, we make a perturbative analysis of the quantum master equation (QME), $\Sigma=0$, for the Wilsonian effective action. It is found that the QME for the UV action determines whether exact gauge symmetry is realized along the renormalization group (RG) flow. The basic task of solving the QME can be reduced to compute the Troost-van Niuwenhuizen-Van Proyen jacobian factor for the classical UV action. When the QME cannot be satisfied, the nonvanishing $\Sigma$ is proportional to a BRS anomaly, which is shown to be preserved along the RG flow. To see how the UV action fulfills the QME in anomaly free theory, we calculate the jacobian factor for a pure Yang-Mills theory in four dimensions.
\end{abstract}

PACS: 11.10Hi; 11.15.Tk; 11.30.-j

Keywords: renormalization group; quantum master equation; Becchi-Rouet-Stora transformation; Ward-Takahashi identity; effective action 


\section{Introduction}

Even if a regularization in field theory is not compatible with a given symmetry, it does not mean that the symmetry is lost. The Wilsonian RG [1] provides us with such an example. Since the approach introduces IR cutoff $k$ to yield the effective action for lower frequency modes, the standard form of gauge symmetry is obviously incompatible with the regularization. Nevertheless, as we have shown in previous papers [3, 4], an effective but exact symmetry can be realized along the RG flow. The key concept for ensuring the presence of the renormalized symmetry is the quantum master equation (QME) in the Batalin-Vilkovisky antifield formalism [5]. The QME for the Wilsonian action of the IR (macroscopic) fields $\Sigma_{k}\left[\Phi, \Phi^{*}\right]=0$ forms a hypersurface in the theory space, ie, the space spanned with coupling constants. An interesting observation is that, once a theory is found on the hypersurface at some IR cutoff, it stays on the hypersurface when we lower the cutoff. In other words, when we have a UV (microscopic) theory satisfying the QME, the QME for the IR theory follows and we have the renormalized symmetry.

In our previous works, we assumed (for anomaly free theory) the existence of a UV action which obeys the QME, $\Sigma\left[\phi, \phi^{*}\right]=0$. It is of course a non-trivial assumption that the QME holds for a UV action. The purpose of this paper is to discuss and justify this assumption within a perturbative framework. In doing this, a UV regularization should be specified. In ref. [4], the UV and IR regularizations were incorporated in a single regulator. Instead, here we treat two regularizations independently and use the PauliVillars (PV) scheme for the UV regularization. This allows us to extract the dependence on the UV regularization out of $\Sigma_{k}\left[\Phi, \Phi^{*}\right]$. Actually, we show that, at the one-loop level, $\Sigma_{k}\left[\Phi, \Phi^{*}\right]$ for the Wilsonian action called the average action becomes $\Sigma\left[\phi_{0}, \phi^{*}\right]$ for some classical field configuration $\phi_{0}$. The latter Ward-Takahashi (WT) operator $\Sigma\left[\phi_{0}, \phi^{*}\right]$ is solely determined by the UV theory and it is, as a functional, independent of the IR cutoff. The IR cutoff comes in only through the classical configuration. Since we may confirm $\Sigma\left[\phi_{0}, \phi^{*}\right]=0$ in an anomaly free theory, we conclude that $\Sigma_{k}\left[\Phi, \Phi^{*}\right]=0$ for any IR cutoff $k$. This demonstrates the presence of the renormalized symmetry along the RG flow.

The UV regularized WT operator $\Sigma\left[\phi, \phi^{*}\right]$ naturally arises when the PV fields are integrated out. For a given classical UV action with the standard BRS symmetry, we may look for a quantum UV action, which solves the QME. The procedure itself is quite straightforward. Our regularized expression for $\Sigma\left[\phi, \phi^{*}\right]$ contains the regularized jacobian factor given by Troost-van Niuwenhuizen-van Proyen (TVV) [6] originally for the calculation of anomalies in the antifield formalism. Since the PV mass terms breaks the standard BRS symmetry, this jacobian factor generates possible "anomaly" terms. In the absence of cohomologically nontrivial anomaly, such "anomaly" terms are all superficial, and should be written as BRS transformation of some local counter terms. Once we find the counter terms, we may satisfy $\Sigma\left[\phi, \phi^{*}\right]=0$. Actually, this task was already done for a pure Yang-Mills theory in ref. [7].

Our formulation should be compared with the so-called fine-tuning procedure [8-10].

\footnotetext{
${ }^{1}$ For recent progress in this subject, see, for example, ref. [2].
} 
There, one fixes gauge non-invariant counter terms to compensate the symmetry breaking terms generated by the regularization, using the effective Ward-Takahashi or SlavnovTaylor identity. In doing this, the UV and IR regularizations were introduced with a single regulator, and the effective WT identity for the Legendre action involving the regulator was analyzed for a fixed IR cutoff $k$. We will see that our formulation has advantages to the fine-tuning on two points. First is the separation of the IR and UV regularizations. Second is the use of the Wilsonian action or the average action, which makes the expression of the WT operator simpler.

Let us emphasize that our formulation applies even to the case that a genuine anomaly is present in the UV theory. In this case, the non-vanishing WT operator $\Sigma\left[\phi_{0}, \phi^{*}\right]$ itself is proportional to the anomaly. Since it is equal to the WT operator $\Sigma_{k}\left[\Phi, \Phi^{*}\right]$ for the Wilsonian action, it implies that the BRS anomaly is preserved along the RG flow.

We also stress that our discussion given in this paper, after a slight modification, is applicable to global symmetries as well.

This paper is organized as follows. In the next section, we briefly summarize some results of our formalism needed to perform subsequent perturbative computation. In section 3, the PV regularization scheme is applied to obtain UV regularized one-loop expressions of the WT operator for the Wilsonian as well as the Legendre effective action. In section 4, the quantum UV action is constructed for a pure Yang-Mills theory, by using the TVV formalism. The last section is devoted to discussion.

\section{The average action and the quantum master equa- tion}

Let us consider a gauge theory and its gauge-fixed action in D-dimensional Euclidean space. All fields including ghosts and those for gauge fixing are denoted collectively by $\phi^{A}$. The index $A$ labels Lorentz indices $\mu, \nu$ of tensor fields, the spinor indices of the fermions, and/or an index distinguishing different types of the generic fields. The Grassmann parity for fields is expressed by $\epsilon\left(\phi^{A}\right)=\epsilon_{A}$. The antifields $\phi_{A}^{*}$ with the opposite Grassmann parity $\epsilon\left(\phi_{A}^{*}\right)=\epsilon_{A}+1$ are introduced to make canonical conjugate pairs

$$
\left(\phi^{A}, \phi_{B}^{*}\right)_{\phi}=\delta_{B}^{A} \equiv \delta_{A B}(2 \pi)^{D} \delta(p-q),
$$

where the antibracket is defined by

$$
\begin{aligned}
(F, G)_{\phi} & \equiv \frac{\partial^{r} F}{\partial \phi^{A}} \frac{\partial^{l} G}{\partial \phi_{A}^{*}}-\frac{\partial^{r} F}{\partial \phi_{A}^{*}} \frac{\partial^{l} G}{\partial \phi^{A}} \\
& =\int \frac{d^{D} p}{(2 \pi)^{D}}\left[\frac{\partial^{r} F}{\partial \phi^{A}(-p)} \frac{\partial^{l} G}{\partial \phi_{A}^{*}(p)}-\frac{\partial^{r} F}{\partial \phi_{A}^{*}(-p)} \frac{\partial^{l} G}{\partial \phi^{A}(p)}\right] .
\end{aligned}
$$

In this paper we use a matrix notation $[$ in which the index $A$ also denotes momentum.

\footnotetext{
${ }^{2}$ For details of this notation, see ref. [4].
} 
Let $S\left[\phi, \phi^{*}\right]$ be a gauge-fixed action. The gauge is fixed by the canonical transformation generated by a gauge fermion. In this gauge-fixed basis, the antifields remain intact. Our formalism is based on a continuum analog of the block-spin transformation [11], where $\left\{\phi_{A}, \phi_{A}^{*}\right\}$ are identified with the UV (microscopic) variables. They are transformed into the IR (macroscopic) variables $\left\{\Phi_{A}, \Phi_{A}^{*}\right\}$ by a coarse-graining procedure. To perform the block-spin transformation, we introduce a test function $f_{k}\left(p^{2}\right)$ for the coarse-graining and cutoff functions $R_{A B}^{k}$. They depend on an IR cutoff $k$. As a matrix, $R_{A B}^{k}$ is invertible? and it may be chosen as

$$
\begin{aligned}
\left(R^{k}\right)_{A B}(p,-q) & =\left(\mathcal{R}^{k}\right)_{A B}(p)(2 \pi)^{D} \delta(p-q), \\
\left(\mathcal{R}^{k}\right)_{A B}(p) & =\frac{\mathcal{\mathcal { R }}_{A B}(p)}{f_{k}\left(1-f_{k}\right)}
\end{aligned}
$$

where $\overline{\mathcal{R}}_{A B}(p)$ are assumed to be polynomials in $p$. The function $f_{k}$ behaves as $f_{k}\left(p^{2}\right) \approx 0$ for $k^{2}<p^{2}$, and $f_{k}\left(p^{2}\right) \approx 1$ otherwise.

We consider a gaussian integral

$$
\begin{aligned}
1=N_{k} \int \mathcal{D} \Phi & \mathcal{D} \Phi^{*} \prod_{A} \delta\left(\Phi_{A}^{*}-f_{k}^{-1} \phi_{A}^{*}\right) \\
& \times \exp \left\{-\frac{1}{2 \hbar}\left(\Phi^{A}-f_{k} \phi^{A}\right) R_{A B}^{k}\left(\Phi^{B}-f_{k} \phi^{B}\right)\right\},
\end{aligned}
$$

with a normalization constant $N_{k}$, and rewrite the path integral of the UV fields.f

$$
\begin{aligned}
Z & =\int \mathcal{D} \phi \mathcal{D} \phi^{*} \prod_{A} \delta\left(\phi_{A}^{*}\right) \exp \left(-S\left[\phi, \phi^{*}\right] / \hbar\right) \\
& =\int \mathcal{D} \Phi \mathcal{D} \Phi^{*} \prod_{A} \delta\left(\Phi_{A}^{*}\right) \exp \left(-W_{k}\left[\Phi, \Phi^{*}\right] / \hbar\right) .
\end{aligned}
$$

Here $W_{k}$ is the Wilsonian effective action, called as the average action [11]. The subtracted average action,

$$
\hat{W}_{k}\left[\Phi, \Phi^{*}\right]=W_{k}\left[\Phi, \Phi^{*}\right]-\frac{1}{2} \Phi^{A} R_{A B}^{k} \Phi^{B},
$$

is the generating functional of the connected cutoff Green functions of the UV fields:

$$
\begin{aligned}
\exp \left(-\hat{W}_{k}\left[\Phi, \Phi^{*}\right] / \hbar\right) & =N_{k} \int \mathcal{D} \phi \mathcal{D} \phi^{*} \prod_{A} \delta\left(\Phi_{A}^{*}-f_{k}^{-1} \phi_{A}^{*}\right) \\
& \times \exp \left\{-\left(S\left[\phi, \phi^{*}\right]+\frac{1}{2} \phi^{A} f_{k}^{2} R_{A B}^{k} \phi^{B}-\Phi^{B} f_{k} R_{B A}^{k} \phi^{A}\right) / \hbar\right\} .
\end{aligned}
$$

In the average action, we find that $\Phi^{A}(p) \approx f_{k}\left(p^{2}\right) \phi^{A}(p)$, ie, the IR fields approximate "the averaged fields." For the antifields, we impose the relation $\Phi_{A}^{*}=f_{k}^{-1} \phi_{A}^{*}$. In (2.7), the

\footnotetext{
${ }^{3}$ The invertible matrix $R_{A B}^{k}$ has the signature $\epsilon\left(R_{A B}^{k}\right)=\epsilon_{A}+\epsilon_{B}$. This matrix and its inverse satisfy $R_{B A}^{k}=(-)^{\epsilon_{A}+\epsilon_{B}+\epsilon_{A} \epsilon_{B}} R_{A B}^{k}$ and $\left(R_{k}^{-1}\right)^{B A}=(-)^{\epsilon_{A} \epsilon_{B}}\left(R_{k}^{-1}\right)^{A B}$.

${ }^{4}$ For simplicity, we suppress the source terms for the UV fields which were included in ref. 团.
} 
terms $\phi^{A} f_{k}^{2} R_{A B}^{k} \phi^{B}$ act as an IR regulator, and the regularization is constructed in such a way that the integration of the UV fields is performed for those modes with momenta larger than $k$. For each UV field $\phi^{A}$, the combination

$$
\Phi^{B} f_{k} R_{B A}^{k} \equiv j_{A}
$$

acts as the source.

The Legendre effective action is given by

$$
\hat{\Gamma}_{k}\left[\varphi, \varphi^{*}\right] \equiv \hat{W}_{k}\left[\Phi, \Phi^{*}\right]+j_{A} \varphi^{A}
$$

where the classical UV fields $\varphi^{A}$ are defined as the expectation values of the UV fields $\phi^{A}$ in the presence of the sources $j_{A}$. The antifields are related each other as

$$
\varphi_{A}^{*} \equiv \phi_{A}^{*}=f_{k} \Phi_{A}^{*}
$$

Another Legendre effective action, directly related to the average action, is given by

$$
\begin{aligned}
\Gamma_{k}\left[\varphi, \varphi^{*}\right] & \equiv \hat{\Gamma}_{k}\left[\varphi, \varphi^{*}\right]-\frac{1}{2} \varphi^{A} f_{k}^{2} R_{A B}^{k} \varphi^{B} \\
& =W_{k}\left[\Phi, \Phi^{*}\right]-\frac{1}{2}\left(\Phi^{A}-f_{k} \varphi^{A}\right) R_{A B}^{k}\left(\Phi^{B}-f_{k} \varphi^{B}\right) .
\end{aligned}
$$

We now discuss how the renormalized BRS symmetry is realized along the RG flow. To this end, we define the WT functional $\Sigma$ for the UV fields:

$$
\Sigma\left[\phi, \phi^{*}\right] \equiv \hbar^{2} \exp (S / \hbar) \Delta_{\phi} \exp (-S / \hbar)=\frac{1}{2}(S, S)_{\phi}-\hbar \Delta_{\phi} S,
$$

where the $\Delta$-derivative is given by

$$
\Delta_{\phi} \equiv(-)^{\epsilon_{A}+1} \frac{\partial^{r}}{\partial \phi^{A}} \frac{\partial^{r}}{\partial \phi_{A}^{*}}=(-)^{\epsilon_{A}+1} \int \frac{d^{D} p}{(2 \pi)^{D}} \frac{\partial^{r}}{\partial \phi^{A}(-p)} \frac{\partial^{r}}{\partial \phi_{A}^{*}(p)} .
$$

We also define the WT operator for the IR fields,

$$
\Sigma_{k}\left[\Phi, \Phi^{*}\right] \equiv \hbar^{2} \exp \left(W_{k} / \hbar\right) \Delta_{\Phi} \exp \left(-W_{k} / \hbar\right)=\frac{1}{2}\left(W_{k}, W_{k}\right)_{\Phi}-\hbar \Delta_{\Phi} W_{k},
$$

where $(,)_{\Phi}$ and $\Delta_{\Phi}$ denote the antibracket and the $\Delta$-derivative for the IR fields. Then, one obtains [4]

$$
\begin{aligned}
\left\langle\Sigma\left[\phi, \phi^{*}\right]\right\rangle_{\phi} \equiv & \hbar^{2} \exp \left(W_{k} / \hbar\right) N_{k} \int \mathcal{D} \phi \mathcal{D} \phi^{*} \prod_{A} \delta\left(f_{k} \Phi_{A}^{*}-\phi_{A}^{*}\right) \\
& \times \exp \left\{-\frac{1}{2 \hbar}\left(\Phi-f_{k} \phi\right)^{A} R_{A B}^{k}\left(\Phi-f_{k} \phi\right)^{B}\right\} \Delta_{\phi} \exp (-S / \hbar) \\
= & \Sigma_{k}\left[\Phi, \Phi^{*}\right] .
\end{aligned}
$$

Therefore, if the UV action satisfies the QME $\Sigma\left[\phi, \phi^{*}\right]=0$, the average action automatically obeys the QME, $\Sigma_{k}\left[\Phi, \Phi^{*}\right]=0$ for any $k$. 
幽,

The WT operator $\Sigma_{k}\left[\Phi, \Phi^{*}\right]$ may be expressed in terms of the Legendre effective action

$$
\Sigma_{k}\left[\Phi, \Phi^{*}\right]=\frac{\partial^{r} \Gamma_{k}}{\partial \varphi^{A}} \frac{\partial^{l} \Gamma_{k}}{\partial \varphi_{A}^{*}}-\hbar f_{k}^{2} R_{A C}^{k}\left(\frac{\partial^{l} \partial^{r} \Gamma_{k}}{\partial \varphi_{C}^{*} \partial \varphi^{B}}\right)\left(\left[f_{k}^{2} R^{k}+\frac{\partial^{l} \partial^{r} \Gamma_{k}}{\partial \varphi \partial \varphi}\right]^{-1}\right)^{B A} .
$$

When applied to a pure Yang-Mills theory, eq. (2.16) reduces to the "modified SlavnovTaylor" identity obtained by Ellwanger [12]. In the next section, we derive perturbative expressions of (2.15) and (2.16) at one-loop level.

\section{The Pauli-Villars regularization}

In the previous section, we presented a brief summary of our formalism with an IR regularization. For perturbative analysis, a UV regularization should also be included. This can be done, as in previous work [4], by taking the test function $f_{k}$ and the cutoff functions $R^{k}$ that depend on both IR and UV cutoffs. Instead, here we use the Pauli-Villars (PV) method discussed by TVV [6], independently of the IR regularization. Application of the method to (2.15) and (2.16) allows us to extract the dependence on the UV regularization of the WT operator $\Sigma_{k}$. This makes our analysis simpler than previous perturbative studies [9, 10].

\subsection{The WT operator for the average action}

Let us make a one-loop evaluation of our relation (2.15). We begin with a UV action

$$
S\left[\phi, \phi^{*}\right]=S^{(0)}\left[\phi, \phi^{*}\right]+\hbar S^{(1)}\left[\phi, \phi^{*}\right],
$$

where $S^{(0)}$ and $S^{(1)}\left[\phi, \phi^{*}\right]$ denote a gauge-fixed classical action and its counter action, respectively. The BRS invariance of the UV theory is expressed as the classical master equation,

$$
\left(S^{(0)}, S^{(0)}\right)_{\phi}=0
$$

For each field $\phi^{A}$ entering in a loop, introduced is a PV partner $\chi^{A}$ which has the same statistics as $\phi^{A}$, but the path integral is formally defined in such a way that a minus sign is produced in loops. For the PV fields $\chi^{A}$, their antifields $\chi_{A}^{*}$ are also introduced. The BRS transformation of the PV sector is defined such that the total measure is invariant, and the massless part of the PV action is invariant. See ref. [6] for more details of the $\mathrm{PV}$ scheme. The PV action is given by

$$
\begin{aligned}
S_{\mathrm{PV}}\left[\chi, \chi^{*}, \phi, \phi^{*}\right] & =S_{\mathrm{PV}}^{(0)}\left[\chi, \chi^{*}, \phi, \phi^{*}\right]+S_{\mathrm{PV}}^{\Lambda}\left[\chi, \chi^{*}, \phi, \phi^{*}\right] \\
S_{\mathrm{PV}}^{(0)} & =\frac{1}{2} \chi^{A} L_{A B} \chi^{B}+\chi_{A}^{*} K_{B}^{A} \chi^{B}+\frac{1}{2} \chi_{A}^{*} M^{A B} \chi_{B}^{*}, \\
S_{\mathrm{PV}}^{\Lambda} & =\frac{1}{2} \Lambda \chi^{A} T_{A B} \chi^{B},
\end{aligned}
$$


where $S_{\mathrm{PV}}^{(0)}$ is the massless action, and $S_{\mathrm{PV}}^{\Lambda}$ is a mass term with invertible matrix $T_{A B}$ which may depend on the UV fields $\phi^{A}$ but not on the antifields $\phi_{A}^{*}$. We take the mass $\Lambda=M^{2}$ for bosons and $\Lambda=M$ for fermions. The matrices needed to specify the massless action are given by

$$
L_{A B}=\frac{\partial^{l}}{\partial \phi^{A}} \frac{\partial^{r} S^{(0)}}{\partial \phi^{B}}, \quad K_{B}^{A}=\frac{\partial^{l}}{\partial \phi_{A}^{*}} \frac{\partial^{r} S^{(0)}}{\partial \phi^{B}}, \quad M^{A B}=\frac{\partial^{l}}{\partial \phi_{A}^{*}} \frac{\partial^{r} S^{(0)}}{\partial \phi_{B}^{*}} .
$$

Let $\Sigma\left[\phi, \phi^{*}, \chi, \chi^{*}\right]$ be the WT operator for the total UV action $S\left[\phi, \phi^{*}\right]+S_{\mathrm{PV}}\left[\chi, \chi^{*}, \phi, \phi^{*}\right]$ :

$$
\begin{aligned}
\Sigma\left[\phi, \phi^{*}, \chi, \chi^{*}\right]= & \frac{1}{2}\left[\left(S+S_{\mathrm{PV}}, S+S_{\mathrm{PV}}\right)_{\phi}+\left(S+S_{\mathrm{PV}}, S+S_{\mathrm{PV}}\right)_{\chi}\right] \\
& -\hbar\left(\Delta_{\phi}+\Delta_{\chi}\right)\left(S+S_{\mathrm{PV}}\right) .
\end{aligned}
$$

We may define the UV regularized WT operator by

$$
\begin{aligned}
\Sigma_{\mathrm{reg}}\left[\phi, \phi^{*}\right] & \equiv\left\langle\Sigma\left[\phi, \phi^{*}, \chi, \chi^{*}\right]\right\rangle_{\chi} \\
& \equiv \frac{\int \mathcal{D} \chi \mathcal{D} \chi^{*} \prod_{A} \delta\left(\chi_{A}^{*}\right) \Sigma\left[\phi, \phi^{*}, \chi, \chi^{*}\right] \exp \left(-S_{\mathrm{PV}}\left[\chi, \chi^{*}, \phi, \phi^{*}\right] / \hbar\right)}{\int \mathcal{D} \chi \mathcal{D} \chi^{*} \prod_{A} \delta\left(\chi_{A}^{*}\right) \exp \left(-S_{\mathrm{PV}}\left[\chi, \chi^{*}, \phi, \phi^{*}\right] / \hbar\right)} .
\end{aligned}
$$

At one-loop order, the expression of this WT operator can be simplified as follows. First, the requirement of a mode-by-mode cancellation between the jacobian factors for $\phi$ and $\chi$ leads to

$$
\left(\Delta_{\phi}+\Delta_{\chi}\right)\left(S+S_{\mathrm{PV}}\right)=0
$$

Second, it follows from (3.2) that

$$
\left[\frac{1}{2}\left(S_{\mathrm{PV}}^{(0)}, S_{\mathrm{PV}}^{(0)}\right)_{\chi}+\left(S_{\mathrm{PV}}^{(0)}, S^{(0)}\right)_{\phi}\right]_{\chi^{*}=0} \propto \chi^{A} \frac{\partial^{l}}{\partial \phi^{A}} \frac{\partial^{r}}{\partial \phi^{B}}\left(S^{(0)}, S^{(0)}\right)_{\phi} \chi^{B}=0
$$

Third, since $\langle\chi \chi\rangle_{\chi} \sim O(\hbar)$, one finds $\left\langle\left(S_{\mathrm{PV}}^{(0)}, S_{\mathrm{PV}}^{(0)}\right)_{\phi}\right\rangle_{\chi} \sim O\left(\hbar^{2}\right)$. Thus, the remaining terms are given by

$$
\left\langle\Sigma\left[\phi, \phi^{*}, \chi, \chi^{*}\right]\right\rangle_{\chi}=\hbar\left(S^{(0)}, S^{(1)}\right)_{\phi}+\left\langle\left(S_{\mathrm{PV}}^{\Lambda}, S^{(0)}\right)_{\phi}+\left(S_{\mathrm{PV}}^{\Lambda}, S_{\mathrm{PV}}^{(0)}\right)_{\chi}\right\rangle_{\chi}
$$

where

$$
\begin{aligned}
& \left(S_{\mathrm{PV}}^{\Lambda}, S^{(0)}\right)_{\phi}=\frac{\Lambda}{2} \chi^{A}(-)^{\epsilon_{B}}\left(T_{A B}, S^{(0)}\right)_{\phi} \chi^{B} \\
& \left(S_{\mathrm{PV}}^{\Lambda}, S_{\mathrm{PV}}^{(0)}\right)_{\chi}=\Lambda \chi^{A} T_{A C} K_{B}^{C} \chi^{B} .
\end{aligned}
$$

The integration of the PV fields gives

$$
\left\langle\left(S_{\mathrm{PV}}^{\Lambda}, S^{(0)}\right)_{\phi}+\left(S_{\mathrm{PV}}^{\Lambda}, S_{\mathrm{PV}}^{(0)}\right)_{\chi}\right\rangle_{\chi}=\Lambda\left\langle\chi^{A}(T \mathcal{K})_{A B} \chi^{B}\right\rangle_{\chi}=-\hbar \Lambda \operatorname{tr}\left(T \mathcal{K}[L+\Lambda T]^{-1}\right)
$$


where

$$
\mathcal{K}_{B}^{A}=K_{B}^{A}+\frac{1}{2}\left(T^{-1}\right)^{A C}(-)^{\epsilon_{B}}\left(T_{C B}, S^{(0)}\right)_{\phi}
$$

We then obtain

$$
\begin{aligned}
& \Sigma_{\mathrm{reg}}\left[\phi, \phi^{*}\right]=\hbar\left(S^{(0)}, S^{(1)}\right)_{\phi}-\hbar\left(\Delta S^{(0)}\right)_{\text {reg }}, \\
& \left(\Delta S^{(0)}\right)_{\text {reg }} \equiv \operatorname{tr}\left(\mathcal{K}[1+\mathcal{O} / \Lambda]^{-1}\right), \quad \mathcal{O} \equiv T^{-1} L .
\end{aligned}
$$

The WT operator for the IR fields $\Sigma_{k}\left[\Phi, \Phi^{*}\right]$ is given by the functional average of (3.13) over the UV fields. Since the $\Sigma_{\text {reg }}$ is proportional to $\hbar$, we do not need to make the $\phi$ integration. Expanding the UV fields around their classical fields $\phi_{0}$ determined by the saddle-point equations, we obtain

$$
\Sigma_{k}\left[\Phi, \Phi^{*}\right]=\Sigma_{\mathrm{reg}}\left[\phi_{0}, \phi^{*}\right]
$$

This relation is our main result and it has important implications to be discussed in the next subsection.

\subsection{The WT operator for the Legendre action}

In order to compare our formalism with the previous fine-tuning analysis [8-10], we discuss regularized expression of the WT identity for the Legendre effective action. The general relation (2.16) suggests that one should obtain the same conclusion as the previous subsection. We derive the one-loop version of (2.16) directly in order to see how the IR regulator can be separated from the WT identity. To this end, we consider the partition function for the regularized version of the subtracted average action,

$$
\begin{aligned}
\hat{Z}\left[j, \phi^{*}\right]=\exp ( & \left.-\hat{W}_{k \Lambda}\left[j, \phi^{*}\right] / \hbar\right)=N_{k} \int \mathcal{D} \phi \mathcal{D} \chi \mathcal{D} \chi^{*} \prod_{A} \delta\left(\chi_{A}^{*}\right) \\
& \times \exp -\left(S_{\mathrm{tot}}\left[\phi, \phi^{*}, \chi, \chi^{*}\right]-j_{A} \phi^{A}\right) / \hbar
\end{aligned}
$$

where the sources $j_{A}$ are related to the IR fields as in (2.8), and the total action is given by

$$
\begin{aligned}
S_{\mathrm{tot}}\left[\phi, \phi^{*}, \chi, \chi^{*}\right] & =S_{k}\left[\phi, \phi^{*}\right]+S_{\mathrm{PV}}\left[\chi, \chi^{*}, \phi, \phi^{*}\right] \\
S_{k}\left[\phi, \phi^{*}\right] & =S\left[\phi, \phi^{*}\right]+\frac{1}{2} \phi^{A} f_{k}^{2} R_{A B}^{k} \phi^{B} .
\end{aligned}
$$

In this subsection, the regularized average action is expressed as $\hat{W}_{k \Lambda}$ to indicate that both the IR and UV regularizations are introduced. The regularized Legendre action, which generates the 1PI cutoff vertex functions of the UV fields, is given by

$$
\hat{\Gamma}_{k \Lambda}\left[\varphi, \varphi^{*}\right]=\hat{W}_{k \Lambda}\left[j, \phi^{*}\right]+j_{A} \varphi^{A}
$$


One obtains the WT identity as is shown in Appendix A:

$$
\begin{aligned}
& \frac{1}{2}\left(\hat{\Gamma}_{k \Lambda}, \hat{\Gamma}_{k \Lambda}\right)_{\varphi} \\
& =\frac{\int \mathcal{D} \phi \mathcal{D} \chi \mathcal{D} \chi^{*} \prod_{A} \delta\left(\chi_{A}^{*}\right) \Xi_{\text {tot }} \exp \left\{-\left(S_{\text {tot }}-\frac{\partial^{r} \hat{\Gamma}_{k}}{\partial \varphi^{A}}(\phi-\varphi)^{A}\right) / \hbar\right\}}{\int \mathcal{D} \phi \mathcal{D} \chi \mathcal{D} \chi^{*} \prod_{A} \delta\left(\chi_{A}^{*}\right) \exp \left\{-\left(S_{\text {tot }}-\frac{\partial^{r} \hat{\Gamma}_{k}}{\partial \varphi^{A}}(\phi-\varphi)^{A}\right) / \hbar\right\}}
\end{aligned}
$$

with the total WT operator

$$
\begin{aligned}
\Xi_{\mathrm{tot}}\left[\phi, \phi^{*}, \chi, \chi^{*}\right] & \equiv \frac{1}{2}\left[\left(S_{\mathrm{tot}}, S_{\mathrm{tot}}\right)_{\phi}+\left(S_{\mathrm{tot}}, S_{\mathrm{tot}}\right)_{\chi}\right]-\hbar\left(\Delta_{\phi}+\Delta_{\chi}\right) S_{\mathrm{tot}} \\
& =\frac{1}{2}\left[\left(S_{\mathrm{tot}}, S_{\mathrm{tot}}\right)_{\phi}+\left(S_{\mathrm{tot}}, S_{\mathrm{tot}}\right)_{\chi}\right] .
\end{aligned}
$$

Here we used the cancellation condition of the jacobian factors (3.7). In contrast to (3.5), the WT operator (3.19) has contributions from the IR regulator.

Let us integrate over the PV fields in (3.18). Neglecting terms of the order $\left\langle O\left(\chi^{4}\right)\right\rangle_{\chi} \sim$ $O\left(\hbar^{2}\right)$, we find that

$$
\begin{aligned}
\left\langle\Xi_{\mathrm{tot}}\right\rangle_{\chi} & =\Xi_{\mathrm{reg}}\left[\phi, \phi^{*}\right]=\sum_{i=1}^{3} \Xi_{\mathrm{reg}}^{\{i\}}\left[\phi, \phi^{*}\right] \\
\Xi_{\mathrm{reg}}^{\{1\}}\left[\phi, \phi^{*}\right] & \equiv \frac{1}{2}\left(S_{k}, S_{k}\right)_{\phi}, \\
\Xi_{\mathrm{reg}}^{\{2\}}\left[\phi, \phi^{*}\right] & \equiv \frac{1}{2}\left\langle\left(\phi^{A} f_{k}^{2} R_{A B}^{k} \phi^{B}, S_{\mathrm{PV}}\right)_{\phi}\right\rangle_{\chi} \\
& =-\frac{\hbar}{2} \phi^{C} f_{k}^{2}\left(R^{k}\right)_{C A}(-)^{\epsilon_{B}}\left(\frac{\partial^{l}}{\partial \phi_{A}^{*}}[D+\Lambda T]_{B E}\right)\left([D+\Lambda T]^{-1}\right)^{B E} \\
& =-\frac{\hbar}{4}\left(\phi^{A} f_{k}^{2} R_{A B}^{k} \phi^{B}, \operatorname{str} \ln [T \mathcal{O}+T \Lambda]\right)_{\phi}, \\
\Xi_{\mathrm{reg}}^{\{3\}}\left[\phi, \phi^{*}\right] & \equiv\left\langle\left(S_{\mathrm{PV}}^{\Lambda}, S^{(0)}\right)_{\phi}+\left(S_{\mathrm{PV}}^{\Lambda}, S_{\mathrm{PV}}^{(0)}\right)_{\chi}\right\rangle_{\chi} \\
& =\Lambda\left\langle\chi^{A}(T \mathcal{K})_{A B} \chi^{B}\right\rangle_{\chi}=-\hbar \operatorname{tr}\left(\mathcal{K}[1+\mathcal{O} / \Lambda]^{-1}\right) .
\end{aligned}
$$

In contrast to the previous subsection, we need to perform the remaining $\phi$ integration to separate the IR regulator:

$$
\begin{aligned}
\frac{1}{2}\left(\hat{\Gamma}_{k \Lambda}, \hat{\Gamma}_{k \Lambda}\right)_{\varphi} & =\frac{\int \mathcal{D} \phi \Xi_{\mathrm{reg}}\left[\phi, \phi^{*}\right] \exp \left\{-\left(S_{k}\left[\phi, \phi^{*}\right]-\frac{\partial^{r} \hat{\Gamma}_{k \Lambda}}{\partial \varphi^{A}}(\phi-\varphi)^{A}\right) / \hbar\right\}}{\int \mathcal{D} \phi \exp \left\{-\left(S_{k}\left[\phi, \phi^{*}\right]-\frac{\partial^{r} \hat{\Gamma}_{k \Lambda}}{\partial \varphi^{A}}(\phi-\varphi)^{A}\right) / \hbar\right\}} \\
& \equiv\left\langle\Xi_{\mathrm{reg}}\left[\phi, \phi^{*}\right]\right\rangle_{\phi} .
\end{aligned}
$$

Making a shift of variables $\phi^{A} \rightarrow \phi^{A}+\varphi^{A}$, and an expansion in powers of $\phi^{A}$, one obtains

$$
\begin{aligned}
\frac{1}{2}\left(\hat{\Gamma}_{k \Lambda}, \hat{\Gamma}_{k \Lambda}\right)_{\varphi} & =\left\langle\Xi_{\mathrm{reg}}\left[\varphi, \varphi^{*}\right]+\phi^{A} \frac{\partial^{l} \Xi_{\mathrm{reg}}}{\partial \varphi^{A}}+\frac{1}{2} \phi^{A} \frac{\partial^{l}}{\partial \varphi^{A}} \frac{\partial^{r} \Xi_{\mathrm{reg}}}{\partial \varphi^{B}} \phi^{B}+\cdots\right\rangle_{\phi} \\
& =\sum_{i=1}^{4} \Xi_{\mathrm{reg}}^{\{i\}}\left[\varphi, \varphi^{*}\right]
\end{aligned}
$$


where $\Xi_{\text {reg }}^{\{i\}}\left[\varphi, \varphi^{*}\right] \quad(i=1,3)$ are the same as those in (3.20) with fields and antifields replaced by $\left\{\varphi, \varphi^{*}\right\}$. There appears a new term from the $\phi$ integration. To lowest order of $\hbar$, it is give by

$$
\begin{aligned}
\Xi_{\mathrm{reg}}^{\{4\}}\left[\varphi, \varphi^{*}\right]= & \frac{\hbar}{2} \frac{\partial^{l}}{\partial \varphi^{A}} \frac{\partial^{r} \Xi_{\mathrm{reg}}^{\{1\}}}{\partial \varphi^{B}}\left(\left[f_{k}^{2} R^{k}+T \mathcal{O}\right]^{-1}\right)^{B A} \\
= & \frac{\hbar}{2} \frac{\partial^{l}}{\partial \varphi^{A}} \frac{\partial^{r}}{\partial \varphi^{B}}\left(\varphi^{C} f_{k}^{2} R_{C E}^{k} \varphi^{E}, S^{(0)}\right)_{\varphi}\left(\left[f_{k}^{2} R^{k}+T \mathcal{O}\right]^{-1}\right)^{B A} \\
= & \hbar\left(f_{k}^{2} R_{A C}^{k} K_{B}^{C}+\frac{1}{2}(-)^{\epsilon} \varphi^{C} f_{k}^{2} R_{C E}^{k} \frac{\partial^{l}}{\partial \varphi_{E}^{*}} D_{A B}\right)\left(\left[f_{k}^{2} R^{k}+T \mathcal{O}\right]^{-1}\right)^{B A} \\
= & \hbar f_{k}^{2} R_{A C}^{k} K_{B}^{C}\left(\left[f_{k}^{2} R^{k}+T \mathcal{O}\right]^{-1}\right)^{B A} \\
& +\frac{\hbar}{4}\left(\varphi^{A} f_{k}^{2} R_{A B}^{k} \varphi^{B}, \operatorname{str} \ln \left[f_{k}^{2} R^{k}+T \mathcal{O}\right]\right)_{\varphi}
\end{aligned}
$$

Combining this with $(\overline{3.20})$ gives

$$
\begin{aligned}
\frac{1}{2}\left(\hat{\Gamma}_{k \Lambda}, \hat{\Gamma}_{k \Lambda}\right)_{\varphi} & =\frac{1}{2}\left(S_{k}, S_{k}\right)_{\varphi}+\hbar f_{k}^{2} R_{A C}^{k} K_{B}^{C}\left(\left[f_{k}^{2} R^{k}+T \mathcal{O}\right]^{-1}\right)^{B A}-\hbar \operatorname{tr}\left(\mathcal{K}[1+\mathcal{O} / \Lambda]^{-1}\right) \\
& +\frac{\hbar}{4}\left(\varphi^{A} f_{k}^{2} R_{A B}^{k} \varphi^{B}, \operatorname{str} \ln \left[f_{k}^{2} R^{k}+T \mathcal{O}\right]-\operatorname{str} \ln [T \mathcal{O}+T \Lambda]\right)_{\varphi}
\end{aligned}
$$

Using

$$
\begin{aligned}
& \Gamma_{k \Lambda}=\hat{\Gamma}_{k \Lambda}-\frac{1}{2} \varphi^{A} f_{k}^{2} R_{A B}^{k} \varphi^{B}=\Gamma^{(0)}+\hbar \Gamma_{k \Lambda}^{(1)} \\
& \Gamma^{(0)}=S^{(0)}, \quad \Gamma_{k \Lambda}^{(1)}=S^{(1)}+\operatorname{str} \ln \left(\left[f_{k}^{2} R^{k}+T \mathcal{O}\right][T \mathcal{O}+T \Lambda]^{-1}\right), \\
& \left(\Delta_{\varphi} S^{(0)}\right)_{\text {reg }}=\operatorname{tr}\left(\mathcal{K}[1+\mathcal{O} / \Lambda]^{-1}\right),
\end{aligned}
$$

one finally obtains

$$
\begin{gathered}
\frac{1}{2}\left(\Gamma_{k \Lambda}, \Gamma_{k \Lambda}\right)_{\varphi}-\hbar f_{k}^{2} R_{A C}^{k}\left(\frac{\partial^{l} \partial^{r} \Gamma^{(0)}}{\partial \varphi_{C}^{*} \partial \varphi^{B}}\right)\left(\left[f_{k}^{2} R^{k}+\frac{\partial^{l} \partial^{r} \Gamma^{(0)}}{\partial \varphi \partial \varphi}\right]^{-1}\right)^{B A} \\
=\Sigma_{\mathrm{reg}}\left[\varphi, \varphi^{*}\right] \equiv \hbar\left(S^{(1)}, S^{(0)}\right)_{\varphi}-\hbar\left(\Delta_{\varphi} S^{(0)}\right)_{\mathrm{reg}} .
\end{gathered}
$$

The rhs is again the regularized WT operator for the UV action.

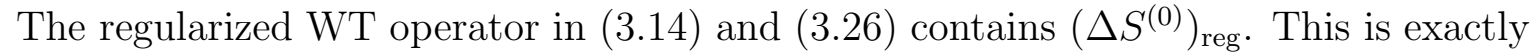
the TVV jacobian factor associated with the BRS transformation in the original gaugefixed UV action. Thus, our main task for solving the QME is to compute the TVV jacobian factor for a given classical UV action. For an anomaly free theory, the TVV jacobian factor becomes a coboundary term, and one can find a local counter action $S^{(1)}$ for which $\left.\Sigma_{\text {reg }}\right|_{\Lambda \rightarrow \infty}=0$. The QME for the UV action is solved in this way. These calculations will be done explicitly for the pure Yang-Mills theory to exemplify the procedure.

When there exists a non-trivial anomaly, such a local counter action cannot be constructed and $\left.\Sigma_{\text {reg }}\right|_{\Lambda \rightarrow \infty}(\neq 0)$ corresponds to the BRS (gauge) anomaly. It follows from $\Sigma_{k}=\left.\Sigma_{\text {reg }}\right|_{\Lambda \rightarrow \infty} \neq 0$ that, when expressed by the classical field configuration, the BRS anomaly does not depend on the IR cutoff and persists along the RG flow. 


\section{The quantum master equation in pure Yang-Mills theory}

Here we construct the quantum UV action for the four-dimensional SU(N) pure YangMills theory based on the TVV formalism given in ref. [6]. The TVV jacobian factor and the local counter terms for cancelling superficial anomaly terms was already calculated in ref. [7]. Since the calculation is somewhat tedious, we give here some results including those obtained at intermediate steps which were not given in ref. [7]. We also retain the contributions from the antifields.

We begin with a gauge-fixed UV action in the Feynman gauge

$$
\begin{array}{r}
S^{(0)}\left[\varphi, \varphi^{*}\right]=-\operatorname{tr} \int d^{4} x\left[\frac{1}{4} F_{\mu \nu}^{2}+\frac{1}{2}(\partial \cdot A)^{2}-\bar{C} \partial \cdot D C\right. \\
\left.+A^{*} \cdot D C+C^{*} C^{2}-\bar{C}^{*} \partial \cdot A\right]
\end{array}
$$

where $B$ fields are eliminated and the trace $\operatorname{tr}$ is over gauge indices. The covariant derivative is given as $D_{\rho} C=\partial_{\rho} C+\left[A_{\rho}, C\right]$. For the fields $\varphi^{A}=\left\{\bar{C}(x), A_{\mu}(x), C(x)\right\}$ and their antifields, the basic matrices we need are given by

$$
\begin{aligned}
L_{A B} & =\frac{\partial^{l}}{\partial \varphi^{A}} \frac{\partial^{r} S}{\partial \varphi^{B}}=\left(\begin{array}{ccc}
0 & \partial_{\nu} C & -\partial \cdot D \\
-C \partial_{\mu} & R_{\mu \nu} & -\left(\partial_{\mu} \bar{C}\right)-A_{\mu}^{*} \\
D \cdot \partial & -\left(\partial_{\nu} \bar{C}\right)-A_{\nu}^{*} & 0
\end{array}\right)(x) \delta(x-y), \\
R_{\mu \nu} & =D_{\mu} D_{\nu}-\delta_{\mu \nu} D^{2}-\partial_{\mu} \partial_{\nu}-2 F_{\mu \nu}, \\
T_{A B} & =\left(\begin{array}{ccc}
0 & 0 & 1 \\
0 & \delta_{\mu \nu} & 0 \\
-1 & 0 & 0
\end{array}\right)(x) \delta(x-y), \\
\mathcal{K}_{A}^{B} & =K_{A}^{B}=\frac{\partial^{l}}{\partial \varphi_{B}^{*}} \frac{\partial^{r} S}{\partial \varphi^{A}}=\left(\begin{array}{ccc}
0 & -\partial_{\mu} & 0 \\
0 & -\delta_{\mu}^{\nu} C & D^{\nu} \\
0 & 0 & C
\end{array}\right)(y) \delta(y-x) .
\end{aligned}
$$

In order to use the heat kernel method, we take the exponential form of the trace,

$$
\begin{aligned}
& \left(\Delta_{\varphi} S^{(0)}\right)_{\text {reg }}=\left(K_{s}\right)_{A}^{B}\left(\exp \left(-\mathcal{O} / M^{2}\right)\right)_{B}^{A} \\
& \quad=\int d^{4} x \int \frac{d^{4} k}{(2 \pi)^{4}} \operatorname{tr}\left[K_{s}(x) \exp (-i k \cdot x) \exp \left(-\mathcal{O}(x) / M^{2}\right)\right] \exp (i k \cdot x),
\end{aligned}
$$

where $\Lambda=M^{2}$ and

$$
\left(K_{s}\right)_{A}^{B}=\frac{1}{2}\left(K-T^{-1} K^{t} T\right)_{A}^{B}=\frac{1}{2}\left(\begin{array}{ccc}
-C & A_{\mu} & 0 \\
0 & 0 & A^{\nu} \\
0 & 0 & C
\end{array}\right)(y) \delta(y-x)=K_{s}(y) \delta(y-x),
$$




$$
\begin{aligned}
\mathcal{O}_{B}^{A}=\left(T^{-1} L\right)_{B}^{A}= & \left(\begin{array}{ccc}
-D \cdot \partial & \left(\partial_{\nu} \bar{C}\right)+A_{\nu}^{*} & 0 \\
-C \partial^{\mu} & R^{\mu}{ }_{\nu} & -\left(\partial^{\mu} \bar{C}\right)-A^{* \mu} \\
0 & \partial_{\nu} C & -\partial \cdot D
\end{array}\right)(x) \delta(x-y) \\
& =\mathcal{O}(x) \delta(x-y),
\end{aligned}
$$

The use of the "symmetrized" matrix [7 $K_{s}$ defined with the transposed matrix $K^{t}$ simplifies our trace calculation. We decompose the matrix $\mathcal{O}(x)$ as

$$
\mathcal{O}(x)=-\left(\partial_{\rho} \mathbf{1}+Y_{\rho}\right) \delta^{\rho \sigma}\left(\partial_{\sigma} \mathbf{1}+Y_{\sigma}\right)-E,
$$

where $1, Y_{\rho}, E$ are $6 \times 6$ matrices. These matrices are given by

$$
\begin{aligned}
& Y_{\rho}=A_{\rho} \mathbf{1}+\frac{1}{2}\left(\begin{array}{ccc}
-A_{\rho} & 0 & 0 \\
C \delta_{\rho}^{\mu} & -A^{\mu} \delta_{\nu \rho}-A_{\nu} \delta_{\rho}^{\mu} & 0 \\
0 & -C \delta_{\rho \nu} & -A_{\rho}
\end{array}\right), \\
& E=\frac{1}{4} A^{2} \mathbf{1}+\left(\begin{array}{ccc}
-\frac{1}{2} \partial \cdot A & -\partial_{\nu} \bar{C}-A_{\nu}^{*} & 0 \\
V^{\mu} & E^{\mu}{ }_{\nu} & \partial^{\mu} \bar{C}+A^{* \mu} \\
C^{2} & -V_{\nu}^{t} & \frac{1}{2} \partial \cdot A
\end{array}\right), \\
& V^{\mu}=-\frac{1}{2} \partial^{\mu} C+\frac{1}{4}\left(3 A^{\mu} C-C A^{\mu}\right), \quad-V_{\nu}^{t}=-\frac{1}{2} \partial_{\nu} C+\frac{1}{4}\left(A_{\nu} C-3 C A_{\nu}\right), \\
& E_{\mu \nu}=\frac{3}{2}\left(\partial_{\mu} A_{\nu}-\partial_{\nu} A_{\mu}\right)+\frac{1}{2} A_{\mu} A_{\nu}-A_{\nu} A_{\mu} .
\end{aligned}
$$

As shown in Appendix B, large $M$ expansion of the momentum integration in (4.4) becomes

$$
\begin{aligned}
& \int \frac{d^{4} k}{(2 \pi)^{4}} \exp (-i k \cdot x) \exp \left(-\mathcal{O}(x) / M^{2}\right) \exp (i k \cdot x) \\
& =-\frac{M^{2}}{(4 \pi)^{2}} \operatorname{tr}(A \cdot \partial C)+\frac{1}{6(4 \pi)^{2}} \operatorname{tr}\left[C \partial^{2} \partial \cdot A-2(\partial C) \cdot A \partial \cdot A+2\left(\partial^{\rho} C\right) A^{\sigma} \partial_{\rho} A_{\sigma}\right. \\
& \left.\quad-2\left(\partial^{\rho} C\right) A^{\sigma} \partial_{\sigma} A_{\rho}+2\left(\partial^{\rho} C\right) A^{\sigma} A_{\rho} A_{\sigma}\right]+O\left(1 / M^{2}\right)
\end{aligned}
$$

We notice that the rhs of (4.8) contains neither the antighosts nor the antifields, and is shown to be a coboundary term. Thus, we find that the QME is satisfied in the $M \rightarrow \infty$ limit

$$
\left(\Delta_{\varphi} S^{(0)}\right)_{\text {reg }}=-\left(S^{(0)}, S^{(1)}\right)_{\varphi}
$$

with the counter actionf

$$
\begin{aligned}
S^{(1)}= & \int d^{4} x \frac{1}{24(4 \pi)^{2}} \operatorname{tr}\left[24 M^{2} A^{2}+\left(\partial_{\mu} A_{\rho}\right)^{2}-3(\partial \cdot A)^{2}-4 A_{\mu}\left(\partial_{\rho} A^{\mu}\right) A^{\rho}\right. \\
& \left.+\left(A^{2}\right)^{2}-3 A_{\mu} A_{\nu} A^{\mu} A^{\nu}\right] .
\end{aligned}
$$

\footnotetext{
${ }^{5}$ The conventional gauge invariant counter terms should be added to construct the total counter action. These terms depend on the renormalization conditions. We do not further discuss this point in this paper.
} 
The finite part of (4.10) is the same as that given in ref. [7].

With the counter action $(4.10), S^{(0)}+\hbar S^{(1)}$ is a UV action satisfying the QME at the one-loop level. It is quite interesting to realize that the IR theory generated from the UV action has the exact renormalized BRS symmetry, though the counter action itself breaks the gauge symmetry.

\section{Discussion}

In our results (3.14) and (3.26), the rhs of these relations can be computed using the UV action, independently of the IR regularization. Therefore, whether the renormalized symmetry along the RG flow exists or not can be determined solely from the UV action. This consequence should be compared with the previous analysis based on the fine-tuning [9, 10]. The fine-tuning analysis corresponds to the computation of the lhs of (3.26) using a single regulator for both IR and UV regularizations for fixed $k$. We would like to emphasize the following advantage of studying the rhs rather than the lhs: The presence of the effective BRS symmetry along the RG flow is guaranteed by properties of the UV action without reference to the IR regularization.

Let us make a remark on application of our formalism to global symmetries such as chiral symmetry. For a given global symmetry, the equations given in the previous section are valid except that ghosts associated with the symmetry are constant. In order to discuss a possible anomaly, we may consider a given theory in a compactified space where the boundary effects can be taken into account. Alternatively, we may introduce space-time dependent ghost fields instead of constant fields. Then, the anomaly is identified with $\left.\Sigma_{\text {reg }}\right|_{\Lambda \rightarrow \infty}$ or its functional derivative with respect to the ghosts.

In the forthcoming paper [13], we apply our formalism to global symmetries, such as the chiral and $\mathrm{SU}(\mathrm{N})$ flavor symmetries, toward our goal to provide a formulation for a non-perturbative study.

\section{Acknowledgments}

This work is supported in part by the Grants-in-Aid for Scientific Research No. 12640258, 12640259, and 13135209 from the Japan Society for the Promotion of Science.

\section{A Derivation of regularized WT identity (3.18)}

The WT identity (3.18) can be derived as follows. We consider the partition function (3.15) with integration variables replaced by

$$
\phi^{\prime A}=\phi^{A}+\left(\phi^{A}, S_{\text {tot }}\right)_{\phi} \lambda
$$




$$
\chi^{\prime A}=\chi^{A}+\left(\chi^{A}, S_{\text {tot }}\right)_{\chi} \lambda,
$$

where $(,)_{\chi}$ is the antibracket with respect to the PV fields, and $\lambda$ is an anticommuting constant. The infinitesimal change of variables leads to

$$
\begin{aligned}
& \int \mathcal{D} \phi \mathcal{D} \chi \mathcal{D} \chi^{*} \prod_{B} \delta\left(\chi_{B}^{*}\right) \exp \left\{-\left(S_{\mathrm{tot}}-j_{C} \phi^{C}\right) / \hbar\right\} \\
\times & \left\{\frac{\partial^{r} S_{\mathrm{tot}}}{\partial \phi^{A}}\left(\phi^{A}, S_{\mathrm{tot}}\right)_{\phi}+\frac{\partial^{r} S_{\mathrm{tot}}}{\partial \chi^{A}}\left(\chi^{A}, S_{\mathrm{tot}}\right)_{\chi}-\hbar\left(\Delta_{\phi}+\Delta_{\chi}\right) S_{\mathrm{tot}}-j_{A}\left(\phi^{A}, S_{\mathrm{tot}}\right)_{\phi}\right\}=0 .
\end{aligned}
$$

The second line apart from the last term is $2 \Xi_{\text {tot }}$. Let us rewrite the contribution from the last term in (A.2) by using the derivatives of the Legendre effective action (3.17):

$$
\begin{aligned}
\frac{\partial^{r} \hat{\Gamma}_{k}}{\partial \varphi^{A}} & =j_{A}\left[\varphi, \varphi^{*}\right], \\
\frac{\partial^{l} \hat{\Gamma}_{k}}{\partial \varphi_{A}^{*}} & =-\hbar \hat{Z}^{-1} \frac{\partial^{l} \hat{Z}}{\partial \varphi_{A}^{*}}+\frac{\partial^{l} j_{B}}{\partial \varphi_{A}^{*}} \varphi^{B},
\end{aligned}
$$

where

$$
\begin{aligned}
\hbar \frac{\partial^{l} \hat{Z}}{\partial \varphi_{A}^{*}}=N_{k} \int & \mathcal{D} \phi \mathcal{D} \chi \mathcal{D} \chi^{*} \prod_{A} \delta\left(\chi_{A}^{*}\right) \exp \left(j_{B} \phi^{B} / \hbar\right) \\
& \times\left\{\frac{\partial^{l} j_{B}}{\partial \varphi_{A}^{*}} \phi^{B}+\hbar \frac{\partial^{l}}{\partial \varphi_{A}^{*}}\right\} \exp \left(-S_{\text {tot }} / \hbar\right) .
\end{aligned}
$$

Then, it follows that

$$
\begin{aligned}
j_{A} \frac{\partial^{l} \hat{\Gamma}_{k}}{\partial \varphi_{A}^{*}} & =-\hbar \hat{Z}^{-1} \int \mathcal{D} \phi \mathcal{D} \chi \mathcal{D} \chi^{*} \prod_{B} \delta\left(\chi_{B}^{*}\right) \exp \left(j_{B} \phi^{B} / \hbar\right) j_{A} \frac{\partial^{l}}{\partial \phi_{A}^{*}} \exp \left(-S_{\text {tot }} / \hbar\right) \\
& =\int \mathcal{D} \phi \mathcal{D} \chi \mathcal{D} \chi^{*} \prod_{B} \delta\left(\chi_{B}^{*}\right) j_{A}\left(\phi^{A}, S_{\text {tot }}\right)_{\phi} \exp \left\{-\left(S_{\text {tot }}-j_{C} \phi^{C}\right) / \hbar\right\} .
\end{aligned}
$$

Using (A.2) and (A.5), one obtains

$$
\frac{1}{2}\left(\hat{\Gamma}_{k}, \hat{\Gamma}_{k}\right)_{\varphi}=\hat{Z}^{-1} \int \mathcal{D} \phi \mathcal{D} \chi \mathcal{D} \chi^{*} \prod_{B} \delta\left(\chi_{B}^{*}\right) \Xi_{\text {tot }} \exp \left\{-\left(S_{\text {tot }}-j_{A} \phi^{A}\right) / \hbar\right\},
$$

which gives (3.18).

\section{B Computation of the TVV jacobian factor (4.8)}

We first consider the momentum integration in (4.4), using the decomposition (4.6) and the notation $\mathcal{D}_{\rho}=\partial_{\rho}+Y_{\rho}$ :

$$
\int \frac{d^{4} k}{(2 \pi)^{4}} \exp (-i k \cdot x) \exp \left(-\mathcal{O}(x) / M^{2}\right) \exp (i k \cdot x)
$$




$$
\begin{aligned}
& =\int \frac{d^{4} k}{(2 \pi)^{4}}\left(\exp \left[\frac{1}{M^{2}}\left(\partial_{\rho}+i k_{\rho}+Y_{\rho}+E\right)\right]\right) \cdot \mathbf{1} \\
& =\int \frac{d^{4} k}{(2 \pi)^{4}} M^{4} \exp \left[-k^{2}+\frac{2 i k^{\rho}}{M} \mathcal{D}_{\rho}+\frac{1}{M^{2}}\left(\mathcal{D}^{2}+E\right)\right] \cdot \mathbf{1} \\
& =M^{2} a(x)+b(x)+O\left(1 / M^{2}\right)
\end{aligned}
$$

where the replacement $k \rightarrow k M$ is made to get the third line, and the large $M$ limit is taken in the last line. Using the Euclidean integral

$$
\int d^{4} k\left(k^{2}\right)^{n} \exp \left(-g k^{2}\right)=\pi^{2}(n+1) ! g^{-(n+2)} \quad(g>0),
$$

we can show that the matrix $a(x)$ takes of the form

$$
\begin{aligned}
a(x) & =\int \frac{d^{4} k}{(2 \pi)^{4}} \exp \left(-k^{2}\right)\left[\frac{(2 i)^{2}}{2 !} k^{\rho} k^{\sigma} \mathcal{D}_{\rho} \mathcal{D}_{\sigma}+\left(\mathcal{D}^{2}+E\right)\right]=\frac{1}{(4 \pi)^{2}}\left(2 \mathcal{D}^{2}+E\right) \\
& =\frac{1}{(4 \pi)^{2}}\left(2 \partial \cdot Y+2 Y^{2}+E\right)
\end{aligned}
$$

This leads to

$$
M^{2} \operatorname{tr}\left(K_{s}(x) a(x)\right)=-\frac{M^{2}}{(4 \pi)^{2}} \operatorname{tr}(A \cdot \partial C),
$$

where the matrix $K_{s}$ given in (4.5) is used, and total derivative terms are ignored. For $b(x)$, we have

$$
\begin{aligned}
b(x)= & \int \frac{d^{4} k}{(2 \pi)^{4}} \exp \left(-k^{2}\right)\left[\frac{(2 i)^{4}}{4 !} k^{\rho} k^{\sigma} k^{\mu} k^{\nu} \mathcal{D}_{\rho} \mathcal{D}_{\sigma} \mathcal{D}_{\mu} \mathcal{D}_{\nu}\right. \\
& \left.+\frac{(2 i)^{2}}{3 !} k^{\rho} k^{\sigma}\left\{\mathcal{D}_{\rho} \mathcal{D}_{\sigma}\left(\mathcal{D}^{2}+E\right)+\mathcal{D}_{\rho}\left(\mathcal{D}^{2}+E\right) \mathcal{D}_{\sigma}+\left(\mathcal{D}^{2}+E\right) \mathcal{D}_{\rho} \mathcal{D}_{\sigma}\right\}+\frac{1}{2 !}\left(\mathcal{D}^{2}+E\right)\right] \\
= & \frac{1}{(4 \pi)^{2}}\left[\frac{1}{6} \mathcal{D}^{\rho} \mathcal{D}^{\sigma}\left(\mathcal{D}_{\rho} \mathcal{D}_{\sigma}-\mathcal{D}_{\sigma} \mathcal{D}_{\rho}\right)+\frac{1}{2} E^{2}+\frac{1}{6}\left(\mathcal{D}^{2} E+E \mathcal{D}^{2}-2 \mathcal{D}^{\rho} E \mathcal{D}_{\rho}\right)\right] \\
= & \frac{1}{(4 \pi)^{2}}\left(\frac{1}{12} W_{\rho \sigma}^{2}+\frac{1}{2} E^{2}+\frac{1}{6} \nabla_{\rho} \nabla^{\rho} E\right) \\
= & \frac{1}{24(4 \pi)^{2}}\left(\begin{array}{ccc}
\alpha & * & * \\
\beta^{\mu} & * & * \\
* & \gamma_{\nu} & \eta
\end{array}\right)(x)
\end{aligned}
$$

where $\nabla^{\rho} E \equiv \partial^{\rho} E+\left[Y^{\rho}, E\right] . \alpha, \beta^{\mu}, \gamma_{\nu}$, and $\eta$ are the matrix elements needed for our trace calculation. Other matrix elements denoted by the asterisks do not contribute to the trace. Actually, we find that

$$
\begin{aligned}
\operatorname{tr}\left(K_{s}(x) b(x)\right) & =\frac{1}{24(4 \pi)^{2}} \operatorname{tr}\left[C(\eta-\alpha)+\left(A_{\mu} \beta^{\mu}+A^{\nu} \gamma_{\nu}\right)\right] \\
& =\frac{1}{12(4 \pi)^{2}} \operatorname{tr}\left(-C \alpha+A_{\mu} \beta^{\mu}\right)
\end{aligned}
$$


because of symmetry property

$$
\operatorname{tr}(C \eta)=-\operatorname{tr}(C \alpha), \quad \operatorname{tr}\left(A_{\mu} \beta^{\mu}\right)=\operatorname{tr}\left(A^{\nu} \gamma_{\nu}\right) .
$$

The matrix elements $\alpha$ and $\beta^{\mu}$ are given by

$$
\begin{aligned}
\alpha & =\alpha\left(W^{2}\right)+6 \alpha\left(E^{2}\right)+2 \alpha\left(\nabla_{\rho} \nabla^{\rho} E\right), \\
\beta^{\mu} & =\beta^{\mu}\left(W^{2}\right)+6 \beta^{\mu}\left(E^{2}\right)+2 \beta^{\mu}\left(\nabla_{\rho} \nabla^{\rho} E\right),
\end{aligned}
$$

where $\alpha\left(W^{2}\right)$, for example, denotes the contribution from the matrix $W^{2}$. We decompose further the last term in (B.5) into

$$
\begin{aligned}
\nabla_{\rho} \nabla^{\rho} E= & \partial^{\rho}\left(\partial_{\rho} E+Y_{\rho} E-E Y_{\rho}\right)+\left[Y^{\rho}, \nabla_{\rho} E\right] \\
= & \partial^{2} E+(\partial \cdot Y) E+2 Y \cdot \partial E-2(\partial E) \cdot Y \\
& -E \cdot \partial Y+Y^{2} E-2 Y^{\rho} E Y_{\rho}+E Y^{2}
\end{aligned}
$$

For the matrices given above, $\alpha$ 's are given by

$$
\begin{aligned}
\alpha\left(W^{2}\right) & =\frac{1}{4}\left(F_{\rho \sigma}-\frac{1}{2}\left[A_{\rho}, A_{\sigma}\right]\right)^{2}, \\
\alpha\left(E^{2}\right) & =\left(\frac{1}{4} A^{2}+\frac{1}{2} \partial \cdot A\right)^{2}-\left(A_{\rho}^{*}+\partial_{\rho} \bar{C}\right) V^{\rho}, \\
\alpha\left(\partial^{2} E\right) & =-\left(\frac{1}{4} \partial^{2} A^{2}+\frac{1}{2} \partial^{2} \partial \cdot A\right), \\
\alpha((\partial \cdot Y) E) & =-\frac{1}{2}(\partial \cdot A)\left(\frac{1}{4} A^{2}+\frac{1}{2} \partial \cdot A\right), \\
\alpha(2 Y \cdot \partial E) & =-A \cdot \partial\left(\frac{1}{4} A^{2}+\frac{1}{2} \partial \cdot A\right), \\
\alpha(-2(\partial E) \cdot Y) & =\left[\partial^{\rho}\left(\frac{1}{4} A^{2}+\frac{1}{2} \partial \cdot A\right)\right] A_{\rho}+\partial \cdot\left(A^{*}+\partial \bar{C}\right) C, \\
\alpha(-E \cdot \partial Y) & =\frac{1}{2}\left(\frac{1}{4} A^{2}+\frac{1}{2} \partial \cdot A\right) \partial \cdot A+\frac{1}{2}\left(A^{*}+\partial \bar{C}\right) \cdot \partial C, \\
\alpha\left(Y^{2} E\right) & =-\frac{A^{2}}{4}\left(\frac{1}{4} A^{2}+\frac{1}{2} \partial \cdot A\right), \\
\alpha\left(-2 Y^{\rho} E Y_{\rho}\right) & =\frac{1}{2} A^{\rho}\left[\left(\frac{1}{4} A^{2}+\frac{1}{2} \partial \cdot A\right) A_{\rho}+\left(A_{\rho}^{*}+\partial_{\rho} \bar{C}\right) C\right], \\
\alpha\left(E Y^{2}\right) & =-\frac{1}{4}\left(\frac{1}{4} A^{2}+\frac{1}{2} \partial \cdot A\right) A^{2}+\frac{1}{4}\left(A_{\rho}^{*}+\partial_{\rho} \bar{C}\right)\left(3 A^{\rho} C-C A^{\rho}\right) .
\end{aligned}
$$

Likewise, we have

$$
\begin{aligned}
\beta^{\mu}\left(W^{2}\right)= & \frac{1}{2}\left(D_{\rho} C \delta_{\sigma}^{\mu}+\frac{1}{2}\left(C A_{\rho}+A_{\rho} C\right) \delta_{\sigma}^{\mu}\right)\left(F_{\rho \sigma}-\frac{1}{2}\left[A_{\rho}, A_{\sigma}\right]\right) \\
& +R^{\mu \nu, \rho \sigma}\left(D_{\rho} C \delta_{\nu \sigma}+\frac{1}{2}\left(C A_{\rho}+A_{\rho} C\right) \delta_{\nu \sigma}\right) \\
\beta^{\mu}\left(E^{2}\right)= & -V^{\mu}\left(\frac{1}{4} A^{2}+\frac{1}{2} \partial \cdot A\right)+\left(E_{\rho}^{\mu}-\frac{A^{2}}{4} \delta_{\rho}^{\mu}\right) V^{\rho}+\left(A^{* \mu}+\partial^{\mu} \bar{C}\right) C^{2},
\end{aligned}
$$




$$
\begin{aligned}
& \beta^{\mu}\left(\partial^{2} E\right)=\partial^{2} V^{\mu}, \\
& \beta^{\mu}((\partial \cdot Y) E)=-\frac{1}{2}\left(\partial^{\mu} C\right)\left(\frac{1}{4} A^{2}+\frac{1}{2} \partial \cdot A\right)+\frac{1}{2}\left(2 \partial \cdot A \delta_{\rho}^{\mu}-\partial_{\rho} A^{\mu}-\partial^{\mu} A_{\rho}\right) V^{\rho}, \\
& \beta^{\mu}(2 Y \cdot \partial E)=-C \partial^{\mu}\left(\frac{1}{4} A^{2}+\frac{1}{2} \partial \cdot A\right)+\left(2 A \cdot \partial \delta_{\rho}^{\mu}-A^{\mu} \partial_{\rho}-A_{\rho} \partial^{\mu}\right) V^{\rho}, \\
& \beta^{\mu}(-2(\partial E) \cdot Y)=-\left(\partial_{\rho} V^{\mu}\right) A^{\rho}-\left[\partial^{\rho}\left(E_{\rho}^{\mu}-\frac{A^{2}}{4} \delta_{\rho}^{\mu}\right)\right] C, \\
& \beta^{\mu}(-E \cdot \partial Y)=-\frac{1}{2} V^{\mu} \partial \cdot A-\frac{1}{2}\left(E^{\mu}{ }_{\nu}-\frac{A^{2}}{4} \delta^{\mu}{ }_{\nu}\right) \partial^{\nu} C \\
& \beta^{\mu}\left(Y^{2} E\right)=\frac{1}{4}\left(3 A^{\mu} C-C A^{\mu}\right)\left(\frac{1}{4} A^{2}+\frac{1}{2} \partial \cdot A\right)+\frac{1}{4}\left(5 A^{2} \delta_{\rho}^{\mu}-4 A_{\rho} A^{\mu}+2 A^{\mu} A_{\rho}\right) V^{\rho}, \\
& \beta^{\mu}\left(-2 Y^{\rho} E Y_{\rho}\right)=\frac{1}{2} C\left[\left(\frac{1}{4} A^{2}+\frac{1}{2} \partial \cdot A\right) A^{\mu}+\left(A^{* \mu}+\partial^{\mu} \bar{C}\right) C\right] \\
& -\frac{1}{2}\left(2 A^{\rho} \delta_{\nu}^{\mu}-A^{\mu} \delta_{\nu}^{\rho}-A_{\nu} \delta^{\mu \rho}\right)\left[V^{\nu} A_{\rho}+\left(E_{\rho}^{\nu}-\frac{A^{2}}{4} \delta_{\rho}^{\nu}\right) C\right] \text {, } \\
& \beta^{\mu}\left(E Y^{2}\right)=\frac{1}{4} V^{\mu} A^{2}-\frac{1}{4}\left(E_{\rho}^{\mu}-\frac{A^{2}}{4} \delta_{\rho}^{\mu}\right)\left(3 A^{\rho} C-C A^{\rho}\right)-\left(A^{* \mu}+\partial^{\mu} \bar{C}\right) C^{2} .
\end{aligned}
$$

In (B.10) and (B.11),

$$
\begin{aligned}
D_{\rho} C= & \partial_{\rho} C+\left[A_{\rho}, C\right], \quad F_{\rho \sigma}=\left[D_{\rho}, D_{\sigma}\right] \\
R_{\rho \sigma}^{\mu \nu}= & {\left[F_{\rho \sigma} \delta^{\mu \nu}-\left(D_{\rho} A^{\mu}-\frac{1}{2} A^{\mu} A_{\rho}\right) \delta_{\sigma}^{\nu}-\left(D_{\rho} A^{\nu}+\frac{1}{2} A_{\rho} A^{\nu}\right) \delta_{\sigma}^{\mu}\right.} \\
& \left.+\frac{1}{2} A^{2} \delta^{\mu}{ }_{\rho} \delta_{\sigma}^{\nu}\right]-[(\rho \leftrightarrow \sigma)] .
\end{aligned}
$$

We then obtain from (B.8), (B.9), (B.10), (B.11) and (B.12)

$$
\begin{aligned}
\operatorname{tr}\left(-C \alpha+A_{\mu} \beta^{\mu}\right)= & 2 \operatorname{tr}\left[C \partial^{2} \partial \cdot A-2(\partial C) \cdot A \partial \cdot A+2\left(\partial^{\rho} C\right) A^{\sigma} \partial_{\rho} A_{\sigma}\right. \\
& \left.-2\left(\partial^{\rho} C\right) A^{\sigma} \partial_{\sigma} A_{\rho}+2\left(\partial^{\rho} C\right) A^{\sigma} A_{\rho} A_{\sigma}\right] .
\end{aligned}
$$

The sum of (B.4) and (B.13) yields (4.8).

\section{References}

[1] K. G. Wilson and J. Kogut, Phys. Rep. C12 (1974), 75.

[2] Proceedings of the second conference on the exact renormalization group, Int. J. of Mod. Phys. A16 (2001) pp. 1809-2136, ed. S. Arnone, Y. Kubyshin, T. Morris and K. Yoshida.

[3] Y. Igarashi, K. Itoh and H. So, Phys. Lett B479 (2000), 336; Prog. Theor. Phys. 104 (2000), 1053. 
[4] Y. Igarashi, K. Itoh and H. So, Prog. Theor. Phys. 106 (2001), 149.

[5] I. A. Batalin and G. A. Vilkovisky, Phys. Lett. 102B (1981), 27.

[6] W. Troost, P. van Niuwenhuizen and A. Van Proeyn, Nucl. Phys. B333 (1990), 727.

[7] F. De Jonghe, R. Siebelink, W. Troost, S. Vandoren, P. van Niuwenhuizen and A. Van Proeyn, Phys. Lett. 289B (1992), 354.

[8] C. Becchi, On the construction of renormalized quantum field theory using renormalization group techniques, in Elementary particles, Field theory and Statistical mechanics, ed. M. Bonini, G. Marchesini and E. Onofri, (Parma University, 1993).

[9] M. Bonini, M. D'Attanasio and G. Marchesini, Nucl. Phys. B418 (1994), 81; B421 (1994), 429; B437 (1995), 163; Phys. Lett. B346 (1995), 87.

M. Bonini and G. Marchesini, Phys. Lett. B389 (1996), 566.

[10] M. Bonini and E. Tricarico, Nucl. Phys. B585 (2000), 253.

M. Bonini, Int. J. Mod. Phys. A16 (2001), 1847.

[11] C. Wetterich, Nucl. Phys. B352 (1991), 529; Phys. Lett. B301 (1993), 90; Z. Phys. C60 (1993), 461.

[12] U. Ellwanger, Phys. Lett. B335 (1994), 364.

[13] Y. Igarashi, K. Itoh and H. So, in preparation. 